\title{
Stromal Predominant Kidney Wilms Tumor
}

National Cancer Institute

\section{Source}

National Cancer Institute. Stromal Predominant Kidney Wilms Tumor. NCI Thesaurus.

Code C9148.

Wilms tumor of the kidney characterized by the predominance of the mesenchymal component. 